\title{
Design and validation of an accelerator for an ultracold electron source
}

\author{
G. Taban, ${ }^{1}$ M. P. Reijnders, ${ }^{1}$ S. C. Bell, ${ }^{2}$ S. B. van der Geer, ${ }^{1}$ O. J. Luiten, ${ }^{1}$ and E. J. D. Vredenbregt ${ }^{1, *}$ \\ ${ }^{1}$ Department of Applied Physics, Eindhoven University of Technology, P.O. Box 513, 5600 MB, Eindhoven, The Netherlands \\ ${ }^{2}$ Centre of Excellence for Coherent X-Ray Science, The University of Melbourne, Victoria 3010, Australia
}

(Received 11 January 2008; published 7 May 2008)

\begin{abstract}
We describe here a specially designed accelerator structure and a pulsed power supply that are essential parts of a high brightness cold atoms-based electron source. The accelerator structure allows a magnetooptical atom trap to be operated inside of it, and also transmits subnanosecond electric field pulses. The power supply produces high voltage pulses up to $30 \mathrm{kV}$, with a rise time of up to $30 \mathrm{~ns}$. The resulting electric field inside the structure is characterized with an electro-optic measurement and with an ion timeof-flight experiment. Simulations predict that $100 \mathrm{fC}$ electron bunches, generated from trapped atoms inside the structure, reach an emittance of $0.04 \mathrm{~mm} \mathrm{mrad}$ and a bunch length of $80 \mathrm{ps}$.
\end{abstract}

DOI: 10.1103/PhysRevSTAB.11.050102

PACS numbers: $67.85 .-\mathrm{d}, 29.20 . \mathrm{Ba}$

\section{BRIGHT ELECTRON SOURCES AND THEIR APPLICATIONS}

Pulsed high brightness electron sources are used, for example, in measuring the temperature of surfaces after interaction with ultrafast lasers [1], in observing transient structure in femtosecond chemistry [2], or in realizing high brightness $\mathrm{x}$-ray sources [3]. The brightest pulsed electron sources are based on the photoemission process to produce electron bunches that are subsequently accelerated in strong electric fields [4].

A way to improve beam brightness is to reduce the source size, because the brightness is proportional to the beam current $I$ divided by the surface area of the beam cross section $\Delta A$, and the solid angle $\Delta \Omega$ associated with the uncorrelated angular spread,

$$
B \sim \frac{I}{\Delta A \Delta \Omega} .
$$

One example of this approach is an electron source based on carbon nanotubes (CNT) field emitters [5]. They are actually the brightest electron sources available at the moment. Here, the electrons are emitted from a submicron surface and are able to produce a current of up to $1 \mu \mathrm{A}$. Some applications, as, for example, ultrafast electron diffraction [6], x-ray free-electron lasers [7], or x-ray production by Compton scattering [8], can also benefit from higher brightness, but require much larger currents than CNTs can provide. In fact, the required currents can only be produced in pulsed mode. For these cases, an alternative route to increasing brightness was proposed [9].

Brightness depends inversely on the square of beam emittance, which in turn depends on the square root of the source temperature $T$,

$$
B \propto \frac{I}{\varepsilon^{2}} \propto \frac{I}{T \cdot \Delta A},
$$

*E.J.D.Vredenbregt@tue.nl where

$$
\varepsilon=\frac{1}{m c} \sqrt{\left\langle x^{2}\right\rangle\left\langle p_{x}^{2}\right\rangle-\left\langle x p_{x}\right\rangle^{2}}
$$

is the so-called root-mean-square (rms) normalized emittance [4]. Here, $m$ is the electron mass, $c$ the speed of light, $x$ the transverse position, $p_{x}$ the transverse momentum, and $\langle\cdots\rangle$ indicates averaging over the entire distribution.

Therefore, if we are able to produce electron bunches with a low initial temperature, emittance will also be low and the brightness high, without having to reduce the source size. In this way, pulsed operation with high peak currents and low emittance can be achieved. Our approach to improve the present brightness of pulsed electron beams is based on this idea of a low temperature source [9]. Here, laser-cooled atoms [10] are ionized just above threshold and an ultracold plasma (UCP) is created [11]. The electrons of this plasma are initially created with a temperature of approximately $1 \mathrm{mK}$. Because of the heating process inside the plasma, the electrons quickly equilibrate to a higher temperature in the order of $10 \mathrm{~K}$, which is still orders of magnitude lower than the electron temperature in photoguns [4].

To prevent a space-charge-induced increase in emittance, high electric fields must be turned on with subnanosecond rise time to bring a beam as fast as possible to sufficiently high energies. It has been shown in Claessens et al. [9] that the brightness of such an electron beam can be orders of magnitude higher than what exists now in the field of (sub)picosecond pulsed electron sources.

In order to achieve the full potential of this type of source, a specialized accelerator structure is required. It combines an atom trap [12] with the possibility to create fast high voltage fields. To this end, we developed a special diode structure together with a pulsed power supply. This article presents the design of both the accelerating structure and pulsed power supply and shows its value as an accelerator for our cold-atom-based electron source. 
It is shown that in this first intermediate setup, ultralow emittances of $0.04 \mathrm{~mm}$ mrad can be achieved in pulsed mode, for bunch charges up to $0.1 \mathrm{pC}$ and $80 \mathrm{ps}$ bunch lengths. The resulting brightness is $\sim 130$ times lower than that of the Linac Coherent Light Source (LCLS) electron source at SLAC [13]. Further improvement of the pulsed high voltage supply, by sharpening the voltage pulse to subnanosecond rise times, should lead to the same emittance, but much shorter pulses of $\sim 0.1 \mathrm{ps}$, resulting in a brightness 10 times higher than the LCLS source. Our final goal is to combine the accelerator presented in this paper with a $1 \mathrm{MV}-0.1 \mathrm{~ns}$ rise time voltage power supply, as proposed in [9]. With that, the source can attain a brightness 30 times higher than the LCLS electron source. Using laser-triggered spark gap technology to switch MV voltages, it is possible to generate $1 \mathrm{~ns}$ long and $1 \mathrm{MV}$ high pulses with $0.1 \mathrm{~ns}$ rise and fall time. As has been shown by several groups, including our own [14], such pulses can be applied across gaps as small as $1 \mathrm{~mm}$ without breakdown, for the simple reason that $1 \mathrm{~ns}$ is too short for a breakdown to occur. The acceleration structure presented in this paper is suited for guiding such voltage pulses. The setup presented here is a first step towards the realization of the electron source concept presented in [9].

\section{ACCELERATOR DESIGN}

A technical drawing of the accelerator is given in Fig. 1. It has a coaxial structure. The advantage of using a coaxial geometry is that it can guide very steep field gradients. The designed structure is tapered to reduce reflections of the incoming electric field. The accelerator consists of an inner conductor on which a negative voltage is applied, and an outer conductor which is grounded. A glass ring is used to support the inner conductor of the structure. The structure is designed such as to allow the trapping of a cloud of cold

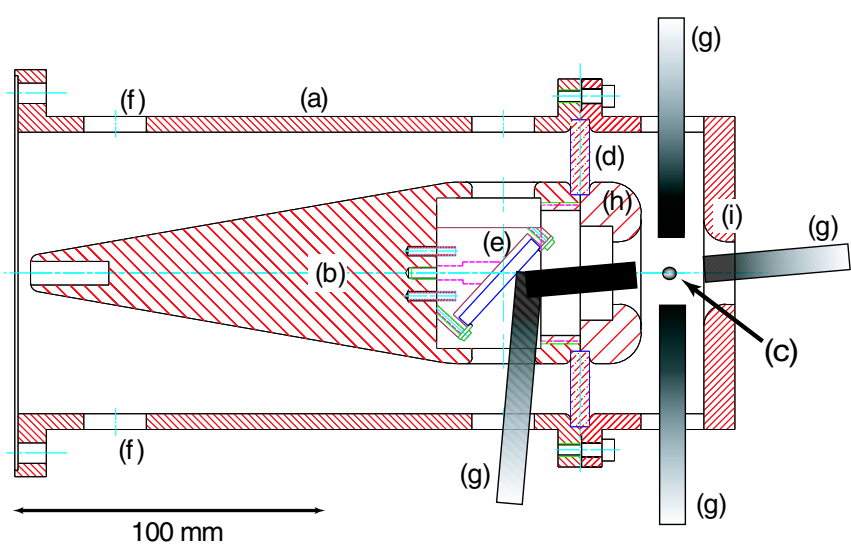

FIG. 1. (Color) Technical drawing of the accelerator (cross section): (a) outer conductor; (b) inner conductor; (c) acceleration point; (d) glass ring; (e) mirror; (f) pumping holes; (g) laser beams; (h) radius of curvature on the inner conductor $R_{i}$; (i) radius of curvature at the acceleration point $R_{a}$. atoms at the center of the accelerating structure, the socalled acceleration point, shown in Fig. 1. The design parameters are given in Table I.

The atom trapping process needs six laser beams [10]. A typical size for the diameter of such a laser beam is $\sim 10 \mathrm{~mm}$. There are six holes of $20 \mathrm{~mm}$ diameter in the outer conductor for the access of these beams. The beams intersect each other in the acceleration point, where the electrons are initially created. One of the laser beams is brought to that point via a mirror placed inside the inner conductor. In addition, there are also holes for the ionizing laser beam and for the electron beam. The inner conductor is connected to a high voltage feedthrough.

In the design process we have maximized the electric field amplitude at the point where the electrons will be initially created. This practically means that the distances between the inner and outer conductor are kept as small as possible. At the same time, the fields must be below the breakdown limit in vacuum, which we conservatively assumed to be $100 \mathrm{kV} / \mathrm{cm}$. For that reason, the distance between the inner and outer conductor is $16 \mathrm{~mm}$, the radius of curvature $R_{i}$ at the end of the inner conductor is $10 \mathrm{~mm}$, and the radius of curvature $R_{a}$ at the acceleration point is $7 \mathrm{~mm}$.

The accelerating structure was first tested with a DC voltage in order to see if it can sustain the maximum DC voltage that it was designed for, namely, $30 \mathrm{kV}$. The metal surface was conditioned by slowly bringing the inner conductor to the maximum operating voltage.

A cylindrically symmetric field map for this structure, calculated with SUPERFISH [15], is shown in Fig. 2. The dimensions of the inner conductor, outer conductor, glass ring and ceramic part of the feedthrough, as listed in Table I, together with their relative permittivity, are used as input. The holes in the outer conductor, which break cylindrical symmetry, are left out. In the figure, the equipotential lines are shown. The electric field strength at the acceleration point is $0.37 \mathrm{kV} / \mathrm{cm}$ per $\mathrm{kV}$ input voltage. The field map is used for analysis and simulations.

In our first experiments we are going to use typical electric field's rise times of $30 \mathrm{~ns}$. The corresponding

TABLE I. Parameters used in the design of the accelerator.

\begin{tabular}{lc}
\hline \hline \multicolumn{1}{c}{ Parameter } & Value \\
\hline Inner conductor maximum outer diameter & $58 \mathrm{~mm}$ \\
Inner conductor length & $205 \mathrm{~mm}$ \\
Outer conductor inner diameter & $90 \mathrm{~mm}$ \\
Outer conductor length & $230 \mathrm{~mm}$ \\
Radius of curvature on the inner conductor $R_{i}$ & $10 \mathrm{~mm}$ \\
Radius of curvature at the acceleration point $R_{a}$ & $7 \mathrm{~mm}$ \\
Glass ring outer diameter & $98 \mathrm{~mm}$ \\
Glass ring inner diameter & $50 \mathrm{~mm}$ \\
Glass ring thickness & $6 \mathrm{~mm}$ \\
Glass ring relative permittivity & 1.7 \\
Ceramic feedthrough relative permittivity & 9 \\
\hline \hline
\end{tabular}




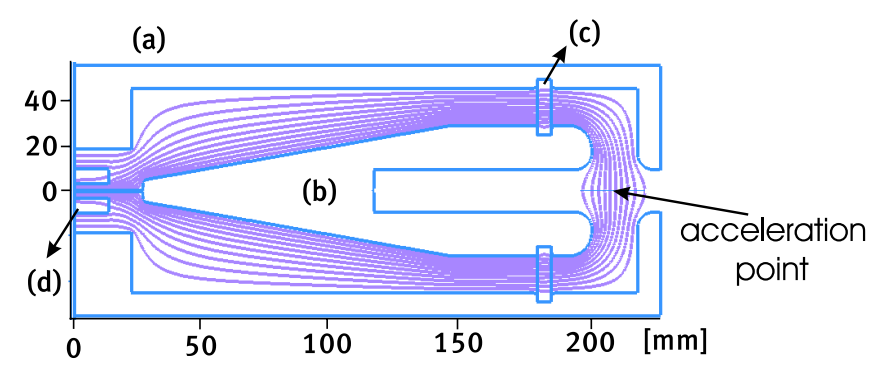

FIG. 2. (Color) Equipotential field lines inside the accelerating structure calculated with SUPERFISH. (a) outer conductor; (b) inner conductor; (c) glass ring; (d) ceramic of the high voltage feedthrough.

wavelength is in the order of meters. This is much larger than the dimensions of the accelerating structure. Therefore, the electric field rises uniformly in the entire structure, not causing reflections. Subsequently, the static map can also be used for a pulsed situation.

The same accelerating structure can be used with even shorter rise time, in the order of $150 \mathrm{ps,}$, which we plan to employ in the future. The wavelength corresponding to subnanosecond rise time becomes comparable with the structure dimensions. In this case, the field can be diminished in amplitude before it reaches the acceleration point due to reflections associated with impedance mismatch. By tapering the inner conductor, the impedance mismatch, and therefore reflections, are minimized.

To check that the combination of the high voltage feedthrough and the accelerator also works at high frequencies, a Hewlett Packard 8753C network analyzer was used. The amplitude and phase of the electric field reflected by the setup were measured as a function of the electromagnetic waves frequency. The frequency interval used in this experiment was between $300 \mathrm{kHz}$ and $1 \mathrm{GHz}$, corresponding to a rise time between $1.2 \mu \mathrm{s}$ and $350 \mathrm{ps}$, respectively. The amplitude of the reflected signal was found to be constant within $0.6 \mathrm{~dB}$ over this range. A near-linear dependence of

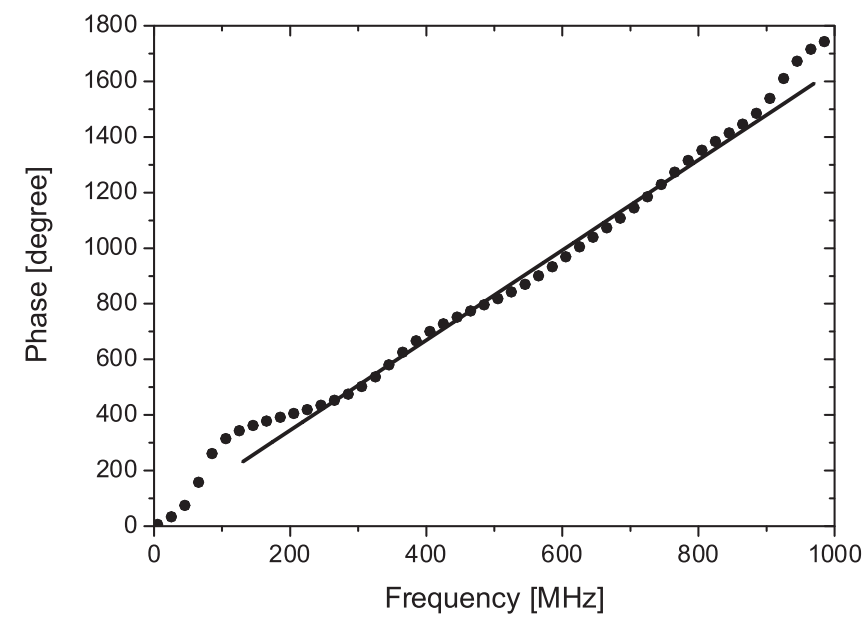

FIG. 3. Phase shift of the reflected signal as given by a network analyzer. the phase lag versus frequency is observed in the 200$900 \mathrm{MHz}$ range (Fig. 3), corresponding to a reflection of the wave at a fixed point located at $0.70 \pm 0.02 \mathrm{~m}$ beyond the point where the network analyzer is connected to the accelerating structure. This is the effective distance that a pulse has to travel from the input on the feedthrough up to the acceleration point, namely, first through the $0.1 \mathrm{~m}$ long feedthrough from a ceramic with $\varepsilon_{r}=9$, second through a $0.2 \mathrm{~m}$ long connection pipe, and third through the $0.2 \mathrm{~m}$ long acceleration. We conclude that up to $350 \mathrm{ps}$ rise time there is no significant distortion of an input high voltage signal.

We have also analyzed the pulse propagation with the 3D time domain solver CST-MICROWAVE STUDIO (MWS).
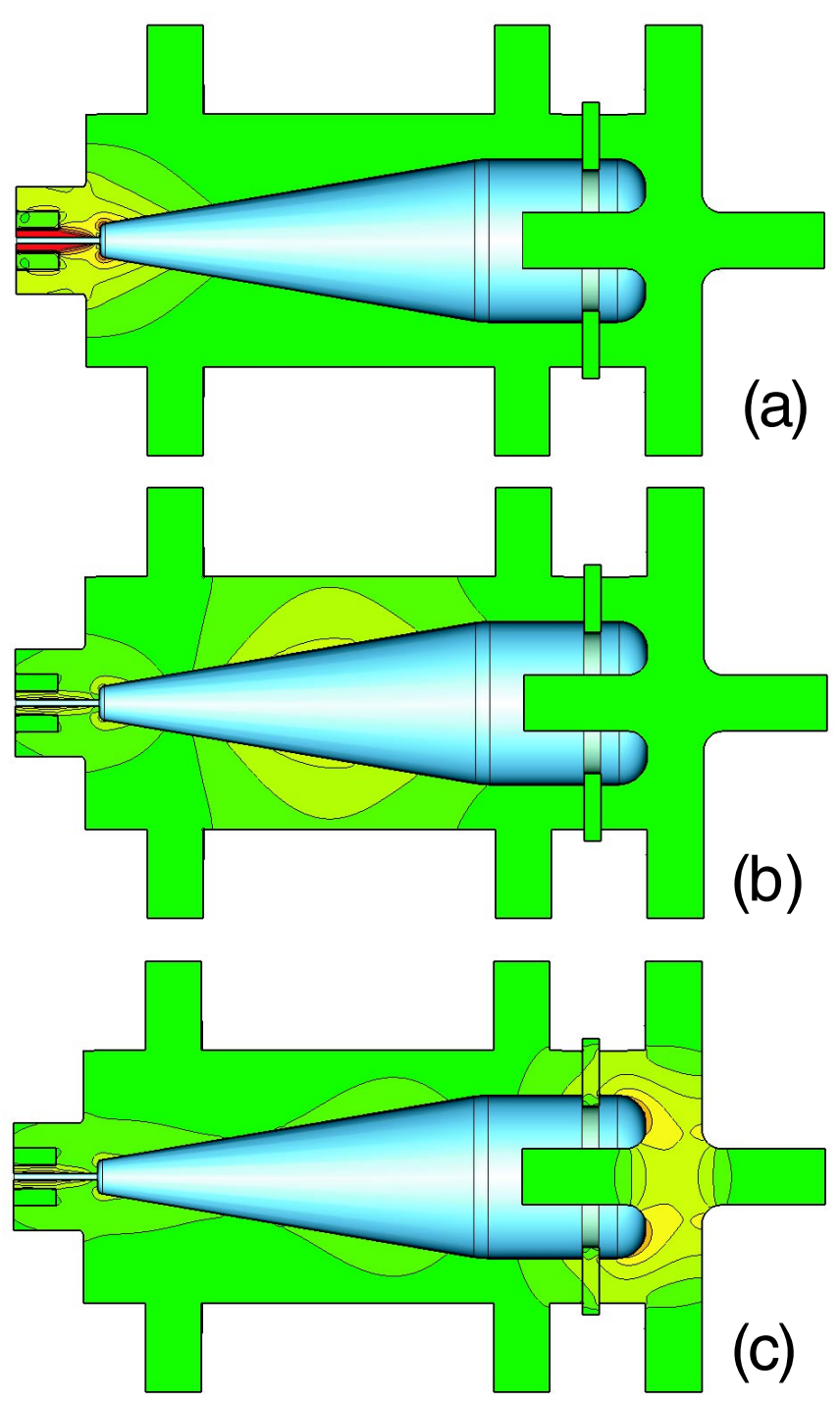

FIG. 4. (Color) CST-MICROWAVE STUDIO simulation for a pulse with a rise time of $150 \mathrm{ps}$. (a) The pulse at the entrance; (b) the pulse halfway through the accelerator; (c) the pulse reaches the acceleration point. In these pictures the vacuum is visualized. The electric field strength is indicated by colors. Red is the maximum electric field strength and green minimum. 


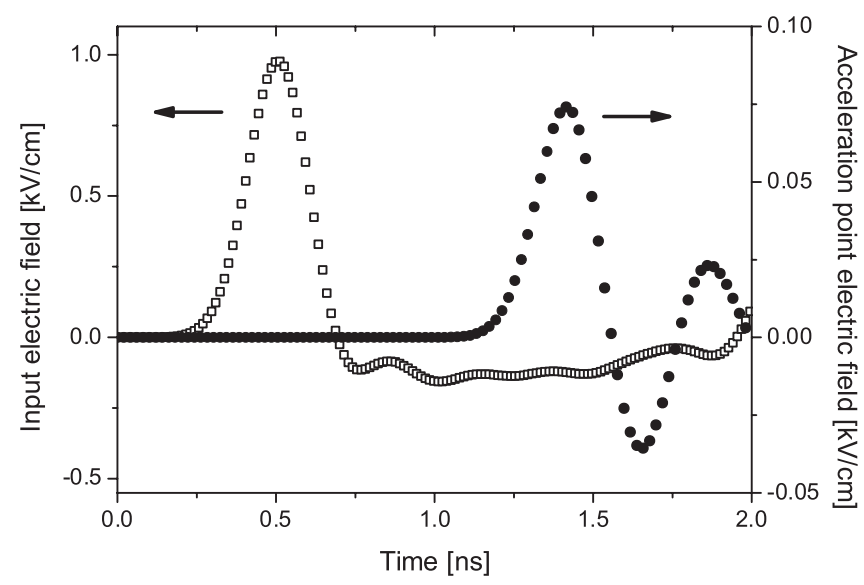

FIG. 5. CST-MICROWAVE STUDIO simulation results for a pulse with a rise time of $150 \mathrm{ps}$. The input electric field is represented by squares $\square$ (left axis) and the electric field at the acceleration point is represented by bullets - (right axis). The maximum electric field at the input was scaled to $1 \mathrm{kV} / \mathrm{cm}$.

An illustration of the process can be seen in Fig. 4. Here, the pulse is shown at three different moments in time: as the pulse enters the structure (a), halfway the structure (b), and as it reaches the acceleration point (c). In this simulation, the access holes in the outer conductor are also included. For 150 ps rise time, we find an electric field strength at the accelerator point of $34 \mathrm{kV} / \mathrm{cm}$ per $\mathrm{kV}$ input voltage, i.e., $8 \%$ decrease compared to SUPERFISH calculations. The rise time behavior at the accelerator entrance and acceleration point was also monitored in the MWS simulations, as shown in Fig. 5. It can be seen from Fig. 5 that the $150 \mathrm{ps}$ rise time of an input pulse remains the same at the acceleration point. The accelerator structure is therefore very well suited for sub-ns risetime voltage pulses.

\section{FAST HIGH VOLTAGE GENERATION}

As it has been stated in our proposal [9], to produce bright electron bunches, a high electric field should be turned on very fast. The proposed source works at a voltage of $1 \mathrm{MV}$ switched on in $150 \mathrm{ps}$, which in principle can be produced with state-of-the-art technology [14]. The required laser-triggered sparkgap technology is however very cumbersome in use and needs further development before it can be applied in practice. In our first experiments we will use commercially available technology to switch $30 \mathrm{kV}$ in tens of nanoseconds. The corresponding fields are high enough to extract bunches up to $1 \mathrm{pC}$ from a UCP, with a very low emittance.

The system used to produce fast and high electric fields consists of two components: a DC high voltage power supply, and a transistor-based switch setup. The high voltage supply unit is a Brandenburg Model 807R. It produces a maximum DC voltage of $30 \mathrm{kV}$ and it delivers a maximum current of $1 \mathrm{~mA}$. The DC power supply is connected via a high voltage coaxial cable to the switch box. The power supply feeds the transistor-based switch. The fast high voltage transistor switch is a Behlke Model HTS 300. It is a solid state switch used to generate high voltage pulses with a very fast leading edge. The maximum operating voltage is $30 \mathrm{kV}$, with a peak current of $30 \mathrm{~A}$. The rise time given in the specifications (with a loading capacitance of $20 \mathrm{pF}$ ) is $15 \mathrm{~ns}$ at a voltage of $24 \mathrm{kV}$. Pulse duration is $150 \mathrm{~ns}$.

To produce the desired rise time of the voltage, the switch is inserted in a classical pulsed discharge circuit (Fig. 6). This means that the energy is collected from a primary energy source (a DC power supply in this case) and is then rapidly released from storage and converted to pulsed form. The output signal goes via a commercial DC ultrahigh-vacuum feedthrough (Kurt J. Lesker Co. Ltd. Model EFT 3012093) into the accelerator structure.

The charging resistor $R_{1}$ is a $40 \mathrm{M} \Omega$ high voltage resistor (Caddock, Type MX485), which limits the current to less than $1 \mathrm{~mA}$. The charging capacitor $C_{1}$ is $2 \mathrm{nF}$. The time constant for the charging circuit is $80 \mathrm{~ms}$. The system can therefore be operated at a repetition rate of a few hertz. After the switch is closed, the charge accumulated on $C_{1}$ is transferred to the accelerator structure, represented by a capacitor $C_{2}$ with $12 \mathrm{pF}$ capacitance. Because of the large difference between the buffer capacitor $C_{1}$ and $C_{2}$, there is only a small decrease in voltage on $C_{1}$ after closing the switch. $R_{2}$ is a $50 \Omega$ current limiting resistor that protects the switch. The time constant for the loading of the accelerator is $R_{2} C_{2} \approx 0.6 \mathrm{~ns}$, so voltage rise time is determined by the rise time of the transistor switch.

The droop time of this circuit is $R_{3} C_{1}=10 \mu \mathrm{s}$. After $150 \mathrm{~ns}$, the switch opens again. The influence of droop on the voltage on $C_{2}$ during the switch closure time is only $2 \%$ of the maximum voltage. At this point the voltage on $C_{2}$ will drop and, together with the leakage resistor $R_{3}$ of $5 \mathrm{k} \Omega$, it will give a decay time constant of $60 \mathrm{~ns}$.

Using a Tektronix high voltage probe (Model P6015A) with a capacitance of $3 \mathrm{pF}$, the output signal given by this switch circuit has been measured on the inner wire of the vacuum feedthrough. A typical voltage-time characteristic is shown in Fig. 7. The negative high voltage pulse first

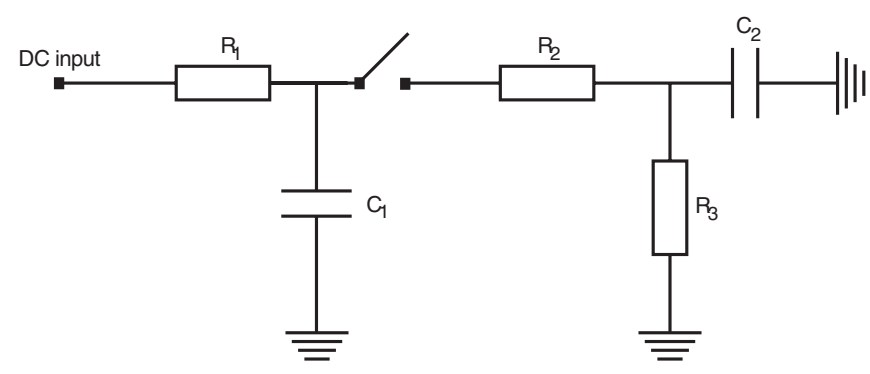

FIG. 6. Schematics of the transistor-based switch used to produce a pulsed high voltage. The values of the components are $R_{1}=40 \mathrm{M} \Omega, R_{2}=50 \Omega, R_{3}=5 \mathrm{k} \Omega, C_{1}=2 \mathrm{nF}$, and $C_{2}=$ $12 \mathrm{pF}$. 


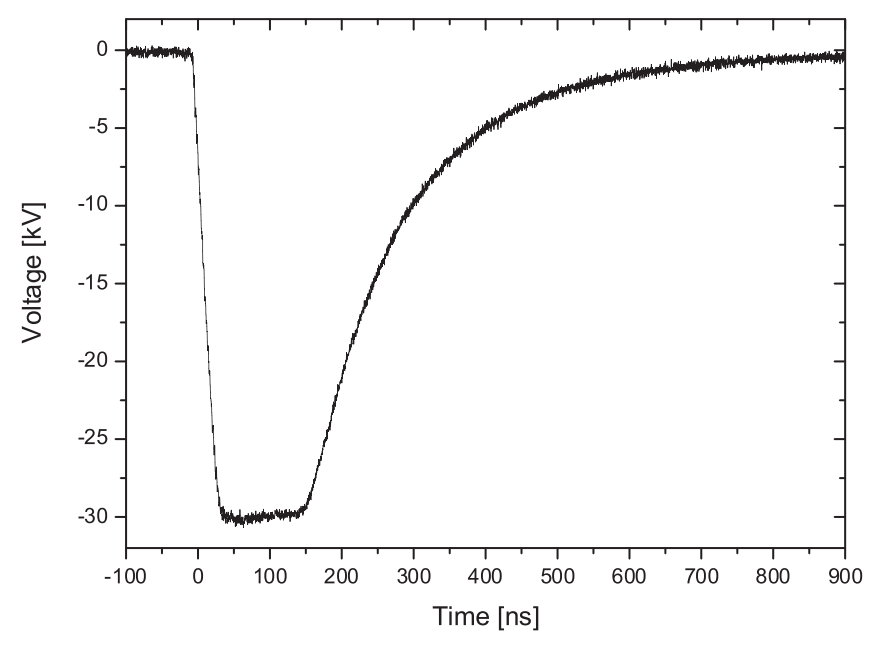

FIG. 7. Output signal $V(t)$ of the switch measured using a Tektronix P6015A high voltage probe.

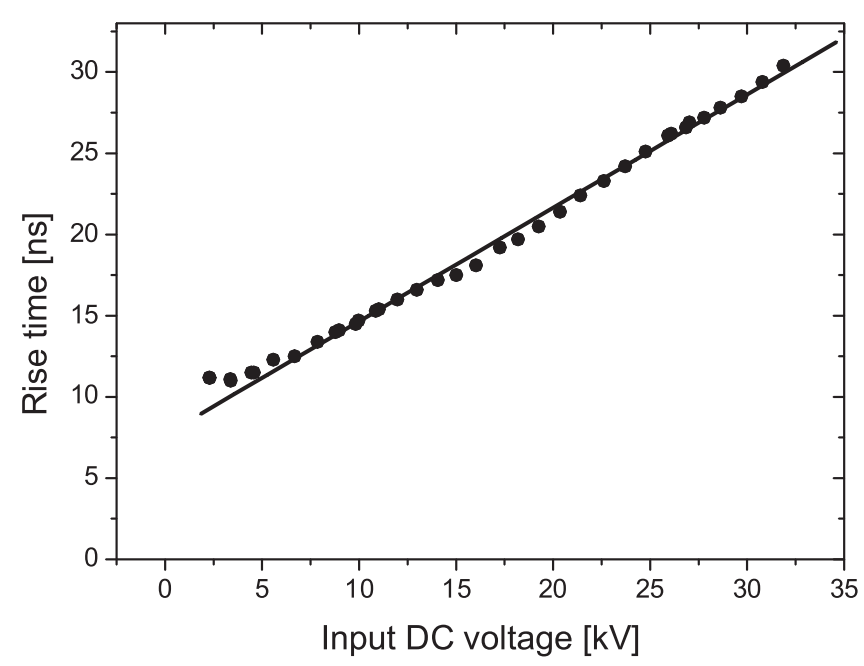

FIG. 8. High voltage pulse rise time at different input DC voltages.

increases linearly, and is followed by a flat top of $150 \mathrm{~ns}$ length, and an exponential decay, in accordance with the design values.

The rise time (defined as 10\%-90\% of voltage amplitude) has been measured as a function of the DC voltage (see Fig. 8). Above $5 \mathrm{kV}$, the rise time depends linearly on voltage, with a slope of $(0.70 \pm 0.01) \mathrm{kV} / \mathrm{ns}$. Accordingly, one gets a rise time of $24.5 \mathrm{~ns}$ at a voltage of $24 \mathrm{kV}$, somewhat larger than the specifications of the switch.

\section{ELECTRIC FIELD MEASUREMENT}

This section deals with static and dynamic measurement of electric fields produced in the accelerating structure.

\section{A. Static electric field measurement with cold ions}

A possibility of measuring the static electric field produced at the acceleration point is by using a time-of-flight
(TOF) method. A cloud of cold atoms is produced at the acceleration point using the same procedure as described in [16]. The cold atoms are subsequently ionized by a pulsed dye laser with a wavelength of $480 \mathrm{~nm}$ and a $6 \mathrm{~ns}$ pulse length. The ionization volume has a cylindrical shape with a radius of $70 \mu \mathrm{m}$ and a height of a few millimeters. The orientation of the cylindrical volume is perpendicular to the acceleration direction. A positive DC voltage is applied to the inner conductor of the accelerator. After the ionization, the ions are accelerated towards a microchannel plate detector placed at a distance $L=282 \mathrm{~mm}$ from the acceleration point. With the help of an oscilloscope triggered by the ionization laser pulse, it is possible to measure the TOF $\tau$ between the photoionization and the moment that the ions reach the detector.

The TOF measurement can be used to calculate the energy $U$ the ions gain in the accelerator:

$$
U=\frac{1}{2} m\left(\frac{L+l_{0}}{\tau}\right)^{2}
$$

where $l_{0}=14.5 \mathrm{~mm}$ corrects for the fact that the ions are accelerated in the first few millimeters and are not immediately at their maximum velocity. The value of $l_{0}$ is determined from simulations. The ionization volume can be precisely moved to different axial positions $z$, within a few millimeters. The TOF measured in this manner gives a $z$-dependency. The $z$-derivative $\frac{1}{q} \frac{d U}{d z}$ of energy is equal to the local electric field.

Figure 9 shows TOF measurements as a function of initial position $z$. Also shown is the ion energy $U$ as a function of $z$ calculated with Eq. (4). The energy $U(z)$ is found to depend approximately linearly on $z$, which allows us to calculate the electric field at the acceleration point: $E(z=0)=0.33 \pm 0.05 \mathrm{~V} / \mathrm{cm}$ per V acceleration voltage, in agreement with the value of $0.37 \mathrm{~V} / \mathrm{cm}$ calculated with the SUPERFISH field map (see Fig. 2). From Fig. 9 can also

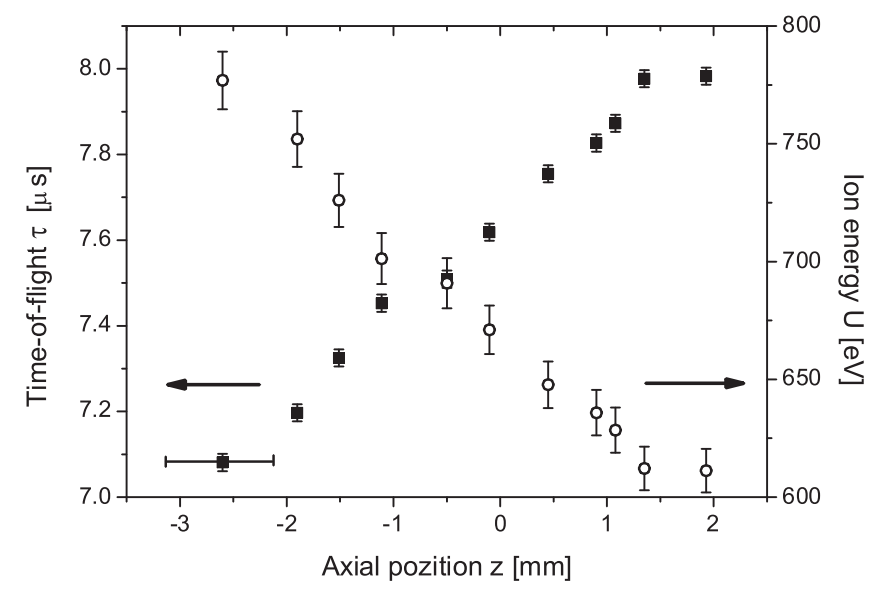

FIG. 9. Ion time-of-flight $\mathbf{\square}$ (left axis) and energy $\bigcirc$ (right axis) versus axial position at an acceleration voltage of $1.36 \mathrm{kV}$. The systematic uncertainty in $z \pm 0.5 \mathrm{~mm}$ is represented for a single point (left bottom). 
be seen that, e.g., at a voltage of $1.36 \mathrm{kV}$, the measured energy $U(0)=665 \mathrm{eV}$ is also, within experimental uncertainty, in agreement with the energy calculated by the electric field integral $\int_{0}^{\infty} e E(z) d z=678 \mathrm{eV}$ from the SUPERFISH field map.

The main source of uncertainty comes from the determination of the axial position $z$ of the ionization volume. In the setup it is determined by two CCD cameras that image the trapped cold gas in two perpendicular directions. This position accuracy is $\pm 0.5 \mathrm{~mm}$. We conclude therefore that the local electric field and the beam energy are in agreement within the experimental accuracy of $15 \%$ with the calculated design values.

\section{B. Pulsed electric field measurement with Pockels effect}

As explained in Sec. III, the rise time of the electric field was measured with the help of a high voltage probe at the exit of the switch box. It should also be checked that there is the same rise time at the accelerating point, where the electrons will be created.

To this purpose, an ellipticity measurement method that employs the Pockels effect was used [17]. The method is based on measuring a change in birefringence induced by an electric field.

A lithium niobate crystal $\left(\mathrm{LiNbO}_{3}\right)$ was used for this purpose. The characteristics of this crystal are given in Table II [18]. Lithium niobate has been chosen because the saturation time, i.e., the time in which an internal electric field builds up and cancels the external electric field, is much longer than the rise times we want to measure, i.e., in the order of ns. ZnTe crystals, for example, are not suitable for this purpose, but can be used for subpicosecond rise times when combined with an ultrafast laser [19].

The setup consists of a HeNe laser with a wavelength of $633 \mathrm{~nm}$, a polarizing beam splitter (PBS) cube, a quarterwave-plate (QWP), another PBS cube, and two photodiodes $\left(P_{1}\right.$ and $\left.P_{2}\right)$ (see Fig. 10). The lithium niobate crystal is placed with the help of a small PVC mount between the high voltage electrodes. The first PBS makes the laser beam linearly polarized. After passing the crystal, it passes through the QWP and becomes circularly polarized. It is subsequently divided by the second PBS. Diodes $P_{1}$ and $P_{2}$ will measure therefore two equal signals when no electric field is present. When a voltage is applied, the electric field induces birefringence and therefore an ellipticity in the

TABLE II. Characteristics of lithium niobate crystal [18].

\begin{tabular}{lc}
\hline \hline \multicolumn{1}{c}{ Parameter } & Value \\
\hline Size and orientation & $5 \times 5 \times 6 \mathrm{~mm} ; z$-cut \\
Electro-optic coefficient $r_{21}$ & $6.5 \mathrm{pm} / \mathrm{V}$ \\
Refractive index $n_{0}$ & 2.3 \\
Relative permittivity $\varepsilon_{r}$ & 85 \\
\hline \hline
\end{tabular}

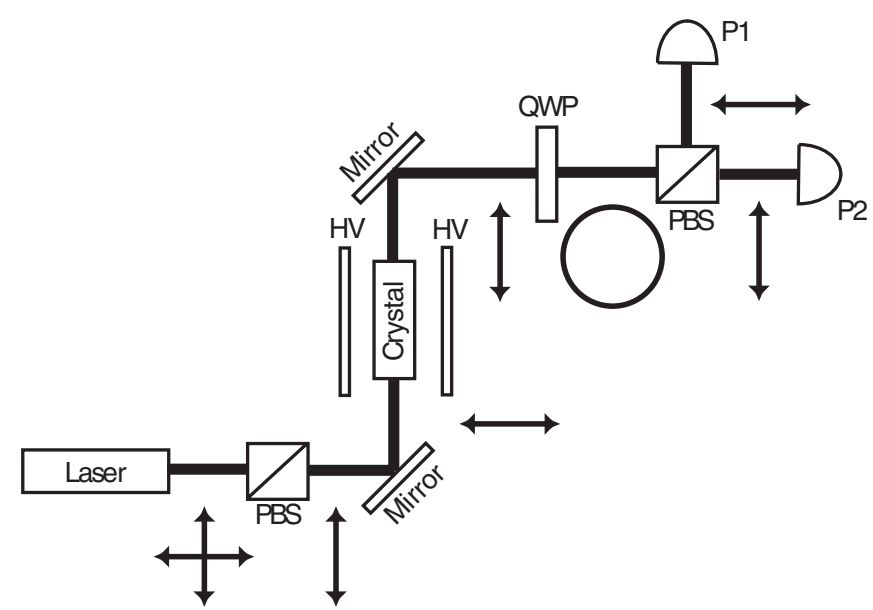

FIG. 10. Drawing of the optical setup used in the electrooptical measurement. PBS is a polarizing beam splitter cube, QWP is a quarter-wave-plate, high voltage electrodes are indicated by $\mathrm{HV}$, and $P_{1}$ and $P_{2}$ are photodiodes. The light polarization is indicated with arrows and a circle.

laser beam polarization, which results in a difference between the two photodiode signals:

$$
\sin (\Delta \varphi)=\frac{I_{1}-I_{2}}{I_{1}+I_{2}}
$$

The phase shift due to the crystal can be written as

$$
\Delta \varphi=\arcsin \left(\frac{2 \pi}{\lambda} r_{21} n_{0}^{3} E d\right)
$$

where $\lambda$ is the laser wavelength, $r_{21}$ the electro-optical coefficient, $n_{0}$ the refractive index, $E$ the internal electric field in the crystal, and $d$ the length that the laser beam travels through in the crystal.

A measurement of the electric field with the crystal placed at the acceleration point is shown in Fig. 11. Here

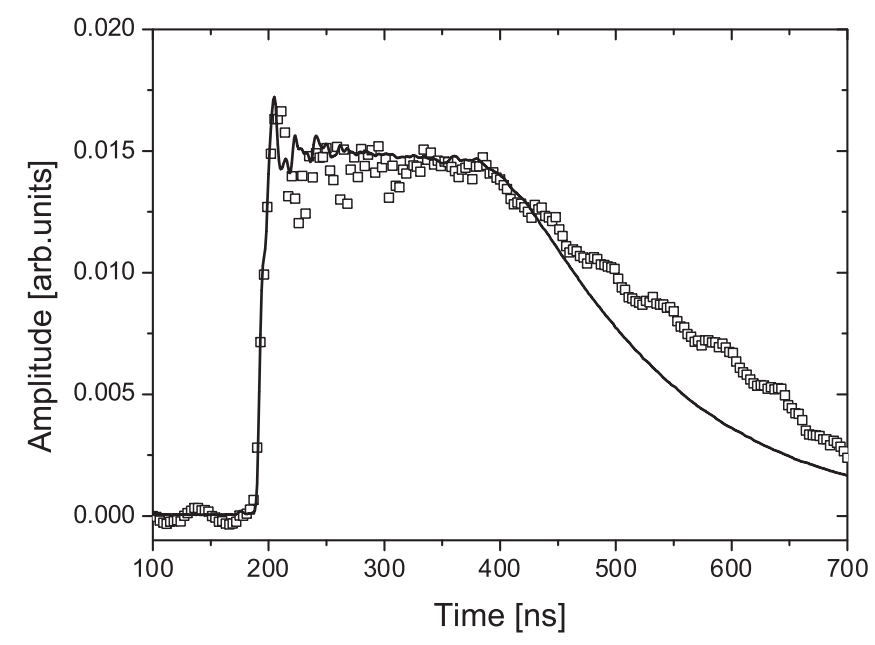

FIG. 11. Electric field measured with electro-optical technique ( $\square$ ) and output of high voltage source measured with high voltage probe (continuous line) placed on the inner conductor of the accelerator. 
is also plotted a signal given by the high voltage probe as measured on the inner conductor of the accelerator. It can be seen that the signal on the inner conductor of the accelerator has the same rise time as the signal measured by the lithium niobate crystal at the acceleration point.

In the quasistatic situation, applying a DC external field of $3 \mathrm{kV}$, a ratio $\left(I_{1}-I_{2}\right) /\left(I_{1}+I_{2}\right)$ of 0.042 was found. Using Eq. (6), this leads to an electric field inside the crystal of $90 \mathrm{~V} / \mathrm{cm}$. A calculation of the electric field with the CST-EM STUDIO simulation program gave $65 \mathrm{~V} / \mathrm{cm}$, which is $30 \%$ lower. In view of the fact that the electro-optic coefficient $r_{21}$ is not very accurately known and the lithium niobate crystal has a strong influence on the field geometry, this is a satisfactory agreement.

\section{VALORIZATION OF THE DESIGN}

As stated in Sec. II, an electric field map was made in SUPERFISH for the DC case (see Fig. 2). By multiplying the electric field of the map with the measured time dependency of the voltage source output (Fig. 11), the map can also be used in the pulsed situation. Fields generated in this way were used in the GPT code to simulate the behavior of an electron bunch accelerated in the designed structure. GPT calculates charged particle trajectories in 3D electromagnetic fields, including all space-charge effects [20].

As initial bunch conditions, exactly the same pancake conditions as in Claessens et al. [9] were chosen, i.e., a radial distribution of $R=2 \mathrm{~mm}$ and a thickness of $15 \mu \mathrm{m}$. The simulations have been performed for two different charges, 100 and $1 \mathrm{fC}$. The high voltage slew rate, which is an essential parameter for our simulations, was $0.7 \mathrm{kV} / \mathrm{ns}$ (see Sec. III). The rms normalized emittance [see Eq. (3)] and the rms bunch length as a function of longitudinal position $z$ obtained from these simulations are shown in Fig. 12.

It can be seen from Fig. 12(a) that the beam has in both situations a very low rms normalized emittance of $0.039 \mathrm{~mm} \mathrm{mrad}(100 \mathrm{fC}$ ) and $0.035 \mathrm{~mm} \mathrm{mrad}(1 \mathrm{fC})$. These remain constant after leaving the accelerating structure. For $100 \mathrm{fC}$, the electron bunch is initially compressed to $\approx 20$ ps due to velocity bunching [9] [Fig. 12(b)] and then expands linearly in time, reaching a length of $80 \mathrm{ps}$ after $300 \mathrm{~mm}$ from the initial point. In case of a smaller charge, $1 \mathrm{fC}$, the velocity bunching effect brings the bunch to a 0.7 ps length. By applying, for example, a pulse of $1 \mathrm{MV}$ in $150 \mathrm{ps}$, a $100 \mathrm{fC}$ bunch can be compressed to $20 \mathrm{fs}$. Clearly, the amount of bunch compression is limited by space-charge forces.

Compared with the LCLS injector at SLAC [13], where electron bunches of $1 \mathrm{nC}$ have been measured with a length of $10 \mathrm{ps}$ and an emittance of $1 \mathrm{~mm}$ mrad, our intermediate setup will be able to deliver a 130 times lower brightness. However, our final configuration of $1 \mathrm{MV}$ in $150 \mathrm{ps}$ will be able to deliver a $100 \mathrm{fC}$ bunch with a 30 times higher brightness.
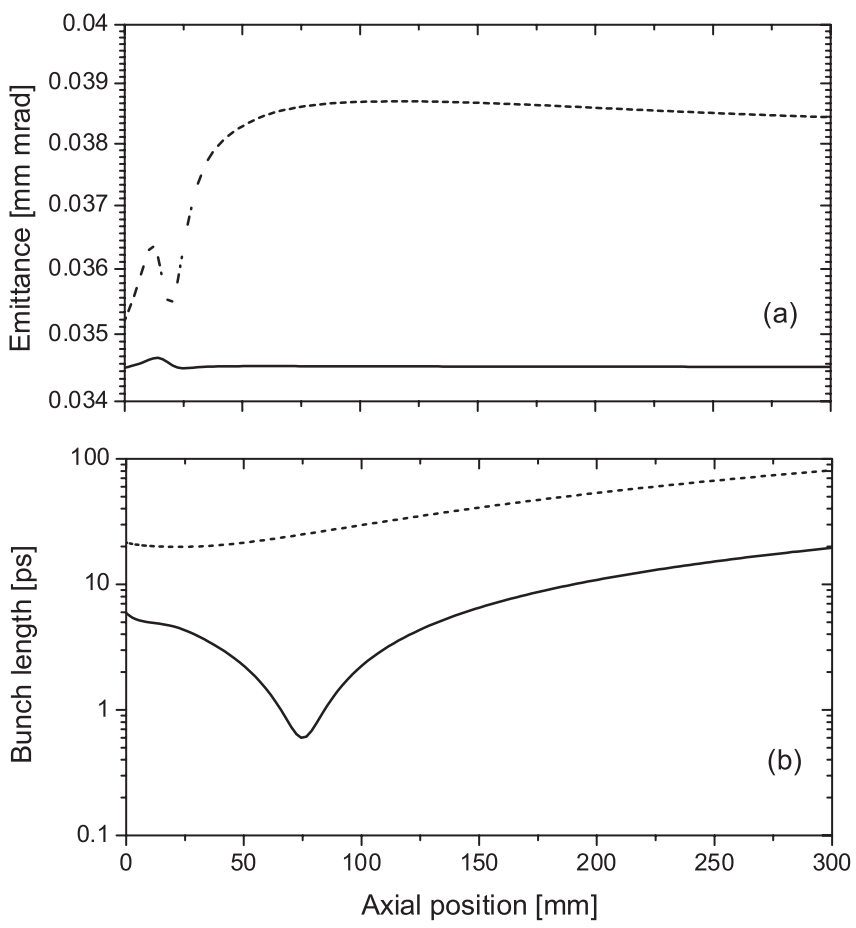

FIG. 12. GPT simulations using a SUPERFISH field map with a slew rate of $0.7 \mathrm{kV} / \mathrm{ns}$ : (a) rms normalized emittance; (b) rms bunch length; both of them as a function of axial position $z$. The dashed line is for $100 \mathrm{fC}$ and the solid line for $1 \mathrm{fC}$.

\section{CONCLUSIONS AND OUTLOOK}

A setup specially developed for a new type of pulsed high brightness electron source based on cold atom traps has been described. It consists of a DC power supply, switch setup, and dedicated accelerator. The electric field rise time has been measured with an electro-optical method. The static electric field at the acceleration point and the energy the accelerated particles gain have also been measured using cold ions TOF measurements. The experimental results and the calculated values agree within the error limits.

Compared with the experiment described in [16], this accelerator has the advantage of being cylindrically symmetric, and provides a higher energy of the electron bunches, making them less sensitive to stray magnetic fields. Simulations show that this setup will be able to produce $100 \mathrm{fC}$ electron bunches with an emittance of $0.04 \mathrm{~mm}$ mrad and a bunch length of $80 \mathrm{ps}$.

For shorter bunch length, an important step is to further reduce the rise time of the electric field. A possible solution is magnetic compression of the high voltage pulse using saturable ferrite cores. In the past, this method produced pulses in the same voltage range with subnanosecond rise time $[21,22]$. The advantage of a shorter rise time is that the beam energy will be higher. Then, the electrons are less sensitive to space-charge effects and the emittance will therefore be even lower. Also the bunch length will be 
smaller due to stronger velocity bunching, leading to higher current and thus higher brightness.

A transmission line transformer (TLT) can be used after the sharpener to increase the amplitude of the high voltage. One specially designed broadband TLT will be available in our group in the future [23]. It is capable of multiplying subnanosecond high voltage pulses by a factor of 10 . In this way, the final goal of $1 \mathrm{MV}-150 \mathrm{ps}$ technology can be reached.

\section{ACKNOWLEDGMENTS}

The authors would like to thank J. A.C. M. van de Ven, A. H. Kemper, L. H. A. M. van Moll, and H. A. van Doorn for their technical support during this project. This work is part of the research program of the Stichting voor Fundamenteel Onderzoek der Materie [FOM, financially supported by the Nederlandse Organisatie voor Wetenschappelijk Onderzoek (NWO)].

[1] H. E. Elsayed-Ali and J. W. Herman, Appl. Phys. Lett. 57, 1508 (1990).

[2] J. Cao, H. Ihee, and A. H. Zewail, Proc. Natl. Acad. Sci. U.S.A. 96, 338 (1999).

[3] R. W. Schoenlein et al., Science 274, 236 (1996).

[4] For an overview, see P. Piot, in The Physics and Applications of High Brightness Electron Beams, edited by J. Rosenzweig, G. Travish, and L. Serafini (World Scientific, Singapore, 2003), p. 127.

[5] N. de Jonge, M. Allioux, J. T. Oostveen, K. B. K. Teo, and W. I. Milne, Phys. Rev. Lett. 94, 186807 (2005).

[6] B. J. Siwick, J. R. Dwyer, R. E. Jordan, and R. J. D. Miller, Science 302, 1382 (2003).

[7] TESLA, the superconducting electron-positron linear collider with an integrated x-ray laser laboratory, DESY technical design report (2001), available from http:// www-hasylab.desy.de/facility/fel.

[8] R. W. Schoenlein et al., C.R. Acad. Sci. Ser. IV 2, 1373 (2001).

[9] B. J. Claessens, S. B. van der Geer, G. Taban, E. J. D. Vredenbregt, and O.J. Luiten, Phys. Rev. Lett. 95, 164801 (2005).

[10] H. J. Metcalf and P. van der Straten, Laser Cooling and Trapping (Springer, Berlin, 1999).

[11] T. C. Killian, S. Kullin, S. D. Bergeson, L. A. Orozco, C. Orzel, and S. L. Rolston, Phys. Rev. Lett. 83, 4776 (1999).

[12] W. Ketterle, K. B. Davis, M. A. Joffe, A. Martin, and D. E. Pritchard, Phys. Rev. Lett. 70, 2253 (1993).

[13] A. Cianchi et al., Phys. Rev. ST Accel. Beams 11, 032801 (2008).

[14] S. Brussaard and D. Vyuga, IEEE Trans. Plasma Sci. 32, 1993 (2004).

[15] J.H. Billen and L. M. Young, POISSON SUPERFISH, Los Alamos National Laboratory Report No. LA-UR-96-1834.

[16] B. J. Claessens, S. B. van der Geer, G. Taban, O. J. Luiten, and E.J.D. Vredenbregt, Phys. Plasmas 14, 093101 (2007).

[17] G. E. Jellison, Jr., C. O. Griffiths, and D. E. Holcomb, Appl. Phys. Lett. 81, 1222 (2002).

[18] A.M. Prokhorov and Y.S. Kuzminov, Physics and Chemistry of Crystalline Lithium Niobate (Adam Hilger, Bristol and New York, 1990).

[19] Q. Wu and X.-C. Zhang, Appl. Phys. Lett. 71, 1285 (1997).

[20] http://www.pulsar.nl/gpt.

[21] J.E. Dolan, H. R. Bolton, and A.J. Shapland, Electron. Lett. 33, 2049 (1997).

[22] N. Seddon and E. Thorton, Rev. Sci. Instrum. 59, 2497 (1988).

[23] D. B. Pawelek, P. A. A. F. Wouters, A. J. M. Pemen, A. H. Kemper, and G.J.H. Brussaard, IEEE Trans. Dielectr. Electr. Insul. 14, 900 (2007). 